\title{
Case Report: Pneumothorax and Pneumomediastinum as Uncommon Complications of COVID-19 Pneumonia-Literature Review
}

\author{
Alvaro Quincho-Lopez, ${ }^{1 \star}$ Dania L. Quincho-Lopez, ${ }^{2}$ and Fernando D. Hurtado-Medina ${ }^{2}$ \\ ${ }^{1}$ San Fernando Medical School, Universidad Nacional Mayor de San Marcos, Lima, Peru; ${ }^{2}$ Diagnostic Imaging Service, Hospital II LNC Luis \\ Negreiros Vega ESSALUD, Lima, Peru
}

\begin{abstract}
As the COVID-19 pandemic progresses, awareness of uncommon presentations of the disease increases. Such is the case with pneumothorax and pneumomediastinum. Recent evidence suggested that these can occur in the context of COVID-19 pneumonia, even in the absence of mechanical ventilation-related barotrauma. We present two patients with COVID-19 pneumonia complicated by pneumomediastinum. The first patient was a 55-year-old woman who developed COVID-19 pneumonia. Her clinical course was complicated by pneumothorax and pneumomediastinum, and, unfortunately, she died 2 days following the admission. The second patient was a 31-year-old man who developed a small pneumomediastinum and was managed conservatively. He had a spontaneous resolution of the pneumomediastinum and was discharged 19 days later. None of our patients required invasive or noninvasive positive pressure ventilation. We performed a literature review of COVID-19 pneumonia cases that developed pneumothorax, pneumomediastinum, or both. The analysis showed that the latter had high mortality (60\%). Thus, it is necessary to pay attention to these complications as early identification and management can reduce the associated morbidity and mortality.
\end{abstract}

\section{INTRODUCTION}

Both pneumothorax and pneumomediastinum are known complications of mechanical ventilation due to intubation. ${ }^{1,2}$ Nonetheless, even without barotrauma involved, pneumothorax or pneumomediastinum, or more rarely both, can be present in the context of COVID-19., ${ }^{3,4}$ Herein, we report two cases of patients infected with SARS-CoV-2, who developed pneumomediastinum, and one of them also presented pneumothorax. We also performed a relevant literature review using the Scopus database.

\section{CASE DESCRIPTIONS}

The first case was a 55-year-old woman with a past medical history of hypertension, uncontrolled bronchial asthma interspersed with periods of inactivity, and morbid obesity. She presented to the emergency department (ED) with 7 days of marked dyspnea, chest pain, and dry cough. Previously, she received outpatient treatment with prednisone and dexamethasone every 8 hours for 5 days. On admission, her vital signs showed tachypnea (22 breaths/minute), with high temperature $\left(38.2^{\circ} \mathrm{C}\right)$, increased heart rate (110 beats/minute), and $85 \%$ saturation. On physical examination, she had bilateral basal crackles and peripheral cyanosis. Laboratory results showed an elevated C-reactive protein (CRP) of $2.66 \mathrm{mg} / \mathrm{dL}$ (normal range $0-0.50 \mathrm{mg} / \mathrm{dL}$ ). Her blood count showed leukocytosis $(30,270$ cells $/ \mu \mathrm{L})$ with a lymphocyte count of 1,210 cells $/ \mu \mathrm{L}$. The patient was reactive to the COVID-19 IgG/lgM rapid test. Non-contrast chest computed tomography (CT) showed some ground-glass opacities of peripheral subpleural location, associated with multiple areas of consolidation in posterior segments of both lower lobes, with the presence of pneumothorax (approximately 20\%) and pneumomediastinum (Figure $1 \mathrm{~A}$ and $\mathrm{B}$ ). She received treatment with azithromycin, ceftriaxone, hydrocortisone, and supplemental oxygen with a reservoir mask. She did not receive noninvasive positive pressure ventilation. The pneumothorax and pneumomediastinum were managed conservatively. However, despite the support measures, the patient died from respiratory failure 2 days after admission.

The second case was a 31-year-old man with a past medical history of chronic gastritis and hypercholesterolemia in control, who presented to the ED with 6 days of dyspnea, general malaise, dry cough, and continuous fever for 4 days. On admission, tachypnea (22 breaths/minute), high temperature $\left(38.5^{\circ} \mathrm{C}\right)$, normal heart rate (94 beats/minute), and saturation of

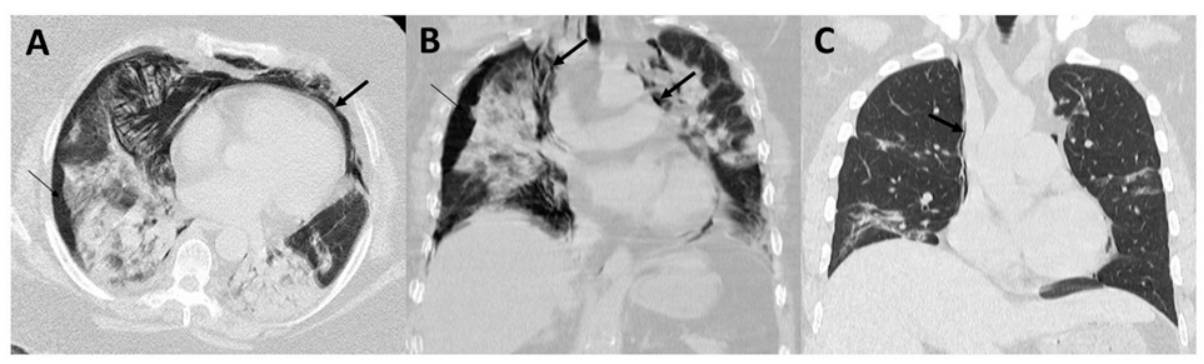

FIGURE 1. (A) Axial image and (B) coronal reconstruction showing pneumothorax (thin arrow) and pneumomediastinum (thick arrow) in case 1. (C) Axial image showing a small pneumomediastinum (thick arrow) in case 2.

\footnotetext{
${ }^{*}$ Address correspondence to Alvaro Quincho-Lopez, San Fernando Medical School, Universidad Nacional Mayor de San Marcos, Jr. Huamanga 165 - apt 704, 15086 Lima, Peru. E-mail: alvaro209.ql@ gmail.com
} 
PNEUMOTHORAX AND PNEUMOMEDIASTINUM IN COVID-19

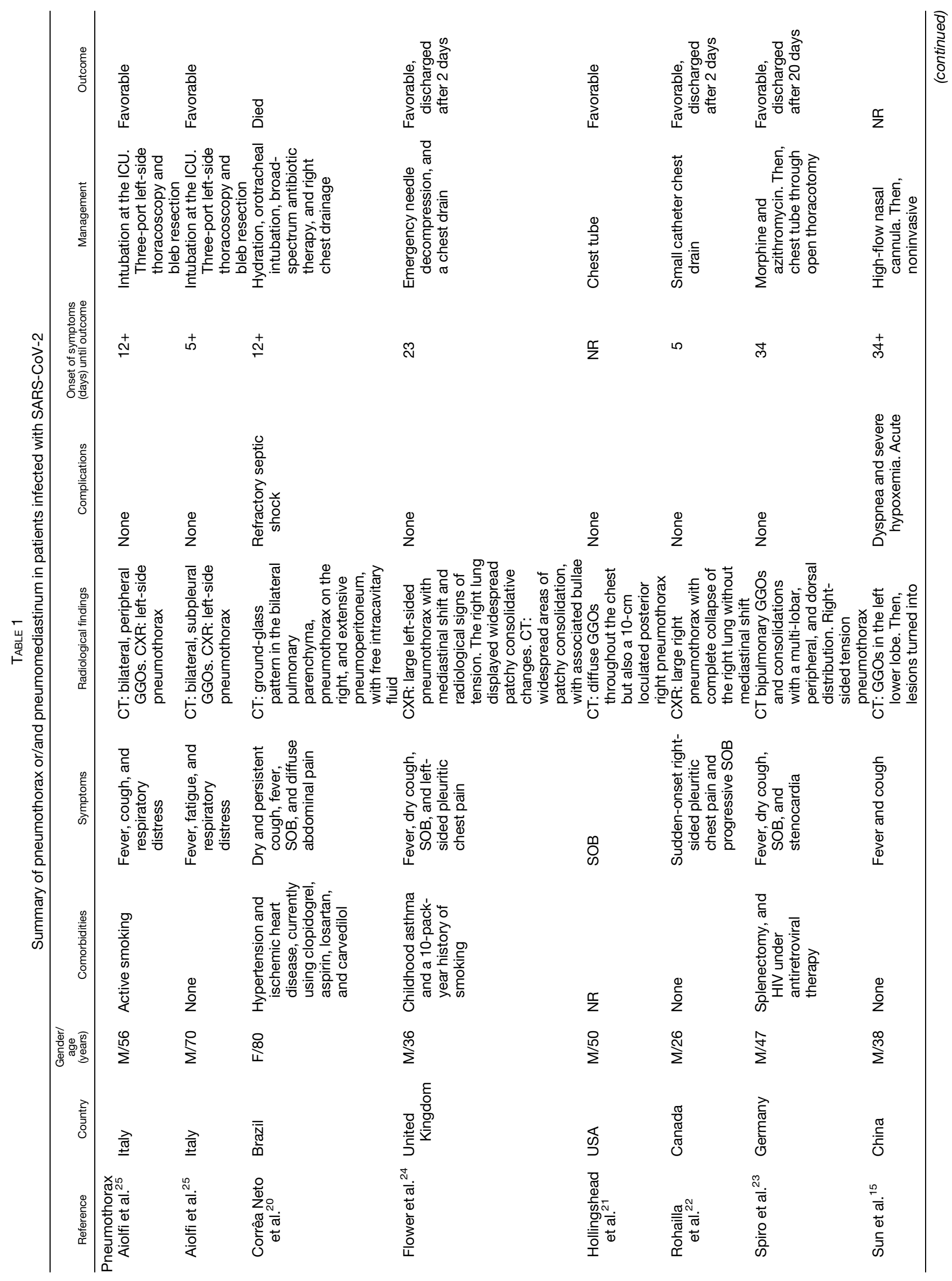




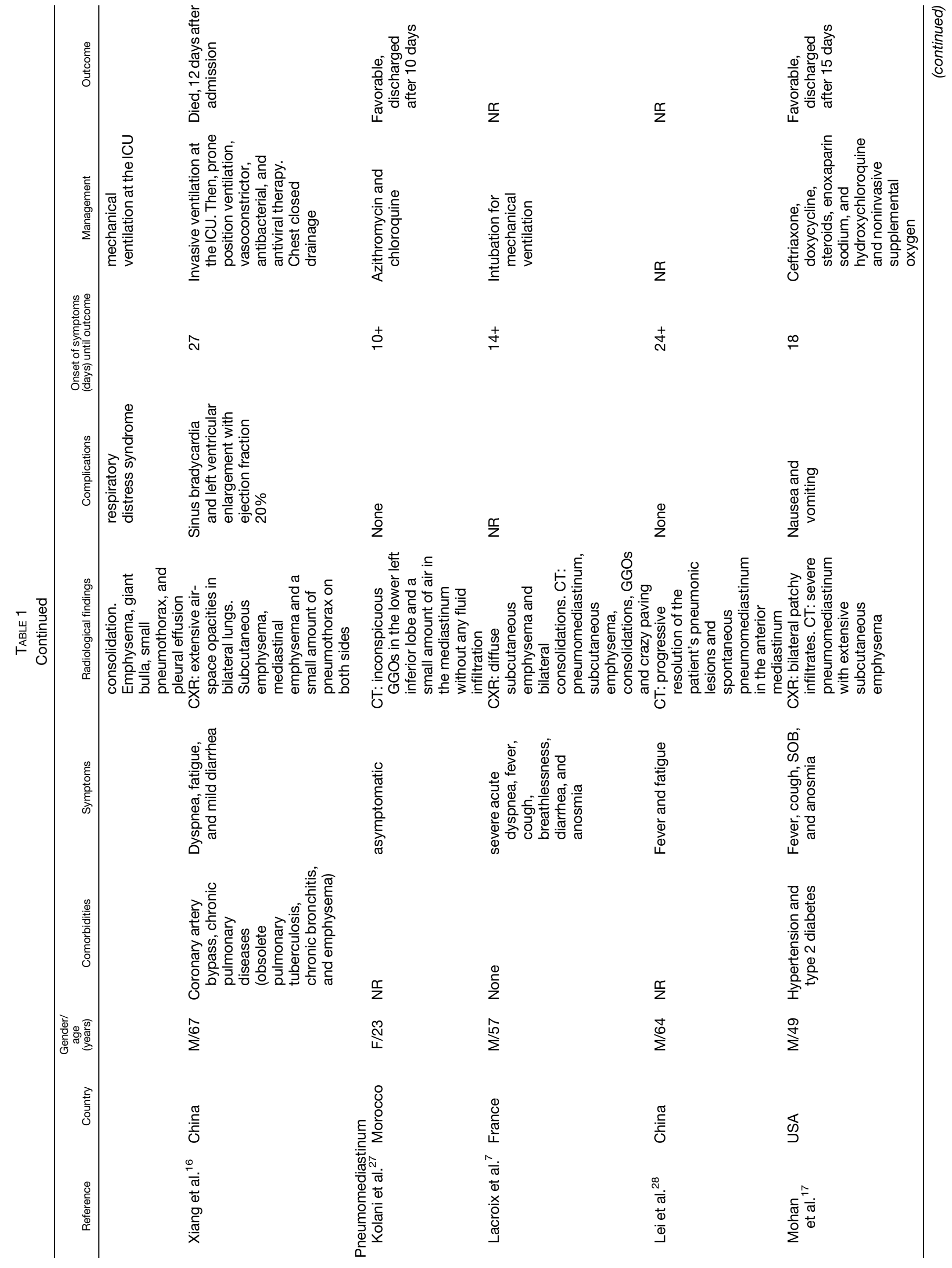




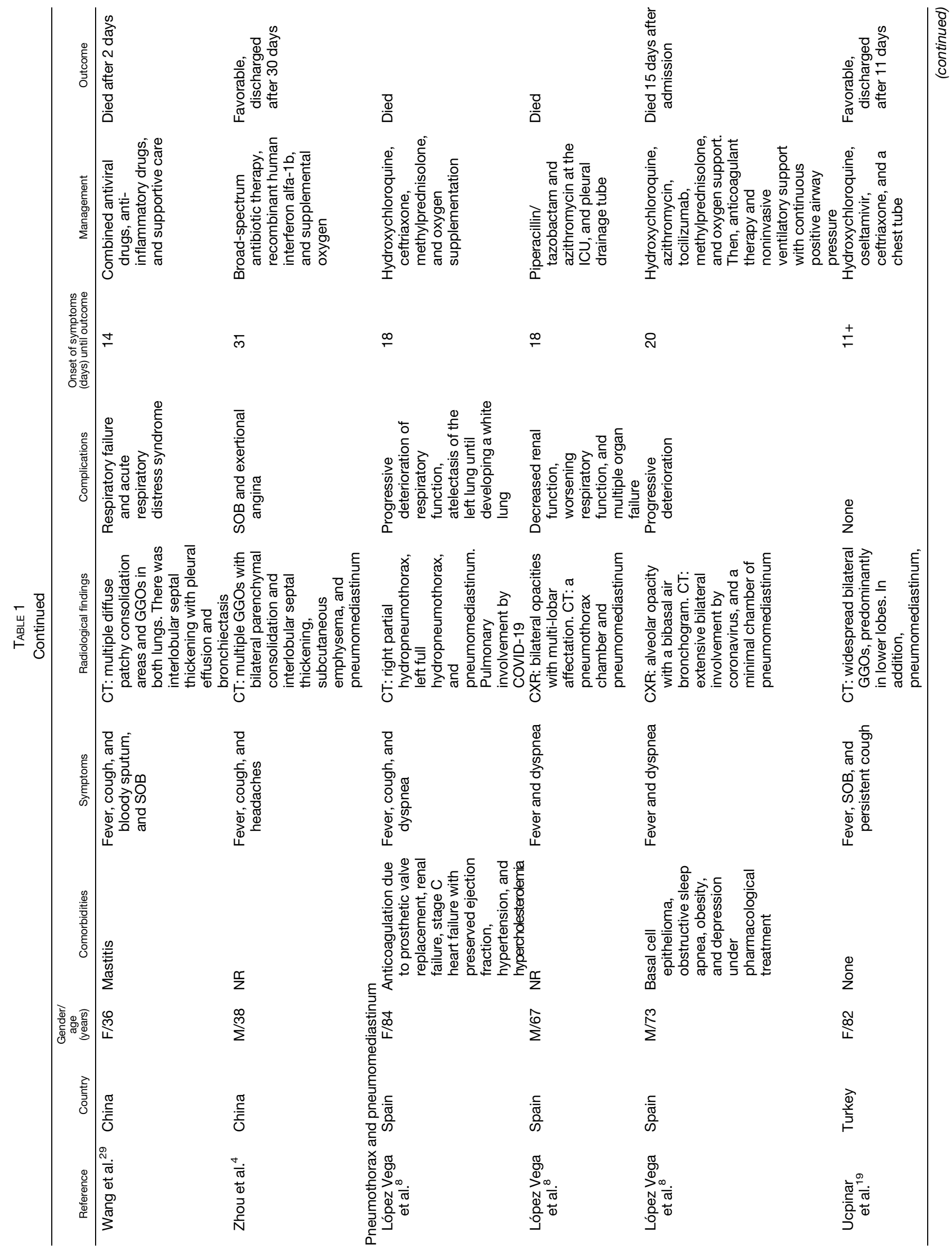


94\% were noted. Physical examination revealed bibasal crackles. He did not receive noninvasive positive pressure ventilation. Laboratory results showed an elevation of CRP of $1.84 \mathrm{mg} / \mathrm{dL}$ (normal range $0-0.50 \mathrm{mg} / \mathrm{dL}$ ). His blood count showed elevated white blood cells $(18,270$ cells $/ \mu \mathrm{L}$ [normal range $3,980-10,040$ cells $/ \mu \mathrm{L}]$ ) with a lymphocyte count of 1,077 cells $/ \mu \mathrm{L}$. The patient was reactive to the $\mathrm{lgG} / \mathrm{lgM}$ rapid test, receiving treatment with azithromycin, ceftriaxone, hydrocortisone, and supplemental oxygen with nasal cannula. A new CT was performed because of desaturation after the removal of oxygen support from the patient (on day 15 of hospitalization) and showed some foci of consolidation in posterior segments of both lower lobes, associated with parenchymal bands in both hemithoraces, with the presence of laminar air content predominantly on the right side, consistent with pneumomediastinum (Figure 1C). The patient remained hospitalized for 19 days. A conservative management was chosen because the pneumomediastinum was very small, and its resolution was observed in the subsequent control after 7 days of discharge.

\section{DISCUSSION}

The symptoms of SARS-CoV-2 infection have been widely characterized in large studies, with fever, cough, and dyspnea being the most frequent. These same studies indicate that only $1-2 \%$ of patients developed pneumothorax ${ }^{5,6}$; although it may occur as the disease progresses, ${ }^{3}$ its presentation is still infrequent, like pneumomediastinum. ${ }^{4}$ The mechanism is not fully elucidated, although it is probably because of rupture of the alveolar wall due to the increasing pressure difference between the alveolus and the pulmonary interstitium. ${ }^{7,8}$

Pneumothorax and pneumomediastinum are defined as the presence of free air in the pleural and mediastinal cavities, respectively. ${ }^{9,10}$ Spontaneous pneumothorax can be primary or secondary, depending on the absence or presence of an underlying lung disease. ${ }^{11}$ By contrast, pneumomediastinum can be primary, or spontaneous, if the cause is idiopathic, or secondary if it responds to a known etiology, whether traumatic or iatrogenic. ${ }^{10}$ Chest pain and dyspnea are the most common symptoms. ${ }^{11,12}$ An important difference is that pneumothorax occurs mainly at rest, ${ }^{9}$ whereas strenuous physical activity has been reported as a triggering event for developing pneumomediastinum. ${ }^{12}$ However, both are more frequent in males. ${ }^{9,12}$ Drug abuse, asthma, and other lung diseases such as chronic obstructive pulmonary disease and interstitial lung disease are some predisposing factors, with tobacco being the most important risk factor in both. ${ }^{9,10}$ None of our patients were active smokers, and only the first one had asthma as a risk factor in developing one of the two complications.

Chest CT as a diagnostic test to identify patients with COVID-19 has a high sensitivity and a high negative predictive value. ${ }^{13}$ Moreover, depending on the lung involvement, it shows different phases: the early phase, where the groundglass pattern of subpleural distribution uni- or bilateral predominates; the progression phase, where, in addition to ground-glass involvement, there are also paved areas or "crazy paving" with diffuse or multi-lobar distribution; the peak, or the most affected phase, where the affected areas progressively consolidate; and, finally, the absorption phase, where ground glass appears secondary to the absorption of consolidations. ${ }^{14}$ 
Table 1 presents a summary of case reports of patients infected with SARS-CoV-2 who presented pneumothorax, pneumomediastinum, or both. Seventeen reports describing 20 patients were found. In total, half of the patients presented a favorable evolution (50\%; 10/20), whereas $30 \%$ $(6 / 20)$ died. Follow-up was not reported in four patients. It is important to note that subcutaneous emphysema was a radiological finding present in 35\% (7/20) of the patients, two in pneumothorax, ${ }^{15,16}$ three in pneumomediastinum, ${ }^{4,7,17}$ and two in both. ${ }^{18,19}$ The treatment for COVID-19 was variable. Nevertheless, for the management of pneumothorax or pneumomediastinum, some patients $(40 \% ; 8 / 20)$ required a chest tube drainage ${ }^{8,16,19-24}$ others $(5 \% ; 1 / 20)$ also required needle aspiration, ${ }^{24}$ and thoracoscopy and bleb resection were required in two (10\%) cases of persistent or recurrent pneumothorax. ${ }^{25}$ The remaining patients were either managed conservatively or not reported.

In those patients with pneumothorax, the majority were male (88.8\%; 8/9), and 55.5\% (5/9) had some comorbidities. Fever was the most frequent symptom on admission (66.6\%; 6/9). Furthermore, the majority presented a favorable evolution (55.5\%; 5/9), with those with the highest number of associated comorbidities having the worst evolution (22.2\%; 2/9). Followup was not reported in one patient. Pneumothorax may also present as a late sequel to COVID-19. ${ }^{21}$ Although most cases report spontaneous pneumothorax, tension pneumothorax is also a possible complication. ${ }^{23,24}$ Bulla associated with pneumothorax is reported in two patients. ${ }^{15,24}$ Some authors consider the rupture of a bulla to be the cause of spontaneous pneumothorax. $^{9}$

Of the pneumomediastinum cases, male gender was the most affected (66.6\%; 4/6), and only 33.3\% (2/6) presented any associated comorbidity. However, in $50 \%$ of the cases, the risk factors were not reported. In $83.3 \%$ (5/6), fever was reported as the most frequent symptom, and one patient did not present any symptoms. The evolution was favorable in $50 \%$ of the cases.

Of the patients who presented pneumothorax and pneumomediastinum at the same time, most of the patients were male $(60 \% ; 3 / 5)$, and $40 \%(2 / 5)$ had some associated comorbidity. Fever was the most frequent symptom (100\%), followed by dyspnea (80\%) and cough (60\%). Death was inevitable in $60 \%(3 / 5)$ of the patients.

Some limitations that we can point out are that certain articles that are not indexed in Scopus could not be included in our review. However, we decided to use Scopus because it contains all the documents included in MEDLINE, ${ }^{26}$ ensuring not only quantity but also quality of the documents. Furthermore, we only considered articles written in English. Our literature review was last updated on July 3 , finding 32 results, of which only 17 are case reports. To enable the reproducibility of our review, we display our search query: (TITLE-ABS \{"2019-nCoV" OR "COVID-19" OR "NCOVID-19" OR "HCoV19" OR "SARS-nCoV" OR "SARS-CoV-2" OR "severe acute respiratory syndrome coronavirus 2"\} OR TITLE-ABS \{coronavirus W/2 [wuhan OR china OR novel OR pneumonia]\}) AND (TITLE-ABS [pneumomediastinum OR pneumothorax]) AND (LIMIT-TO [PUBYEAR, 2020]).

In conclusion, pneumothorax and pneumomediastinum are possible complications of COVID-19 pneumonia, causing acute decompensation that can worsen the prognosis of patients, especially those with underlying lung diseases.
Received July 7, 2020. Accepted for publication July 16, 2020.

Published online July 23, 2020.

Acknowledgment: Publication charges for this article were waived due to the ongoing pandemic of COVID-19.

Financial support: This study was self-financed.

Disclosure: All patients provided consent to share their cases.

Authors' addresses: Alvaro Quincho-Lopez, San Fernando Medical School, Universidad Nacional Mayor de San Marcos, Lima, Peru, E-mail: alvaro209.q|@gmail.com. Dania L. Quincho-Lopez and Fernando D. Hurtado-Medina, Diagnostic Imaging Service, Hospital II LNC Luis Negreiros Vega ESSALUD, Lima, Peru, E-mails: danita.lizzi.mc@gmail.com and fernando1979sf@hotmail.com.

This is an open-access article distributed under the terms of the Creative Commons Attribution (CC-BY) License, which permits unrestricted use, distribution, and reproduction in any medium, provided the original author and source are credited.

\section{REFERENCES}

1. Yao W et al., 2020. Emergency tracheal intubation in 202 patients with COVID-19 in Wuhan, China: lessons learnt and international expert recommendations. $\mathrm{Br} J$ Anaesth 125: e28-e37.

2. Jacobi A, Chung M, Bernheim A, Eber C, 2020. Portable chest $X$-ray in coronavirus disease-19 (COVID-19): a pictorial review. Clin Imaging 64: 35-42.

3. Salehi S, Abedi A, Balakrishnan S, Gholamrezanezhad A, 2020. Coronavirus disease 2019 (COVID-19): a systematic review of imaging findings in 919 patients. AJR Am J Roentgenol 215: 87-93.

4. Zhou C, Gao C, Xie Y, Xu M, 2020. COVID-19 with spontaneous pneumomediastinum. Lancet Infect Dis 20: 510.

5. Chen $\mathrm{N}$ et al., 2020. Epidemiological and clinical characteristics of 99 cases of 2019 novel coronavirus pneumonia in Wuhan, China: a descriptive study. Lancet 395: 507-513.

6. Yang $X$ et al., 2020. Clinical course and outcomes of critically ill patients with SARS-CoV-2 pneumonia in Wuhan, China: a single-centered, retrospective, observational study. Lancet Respir Med 8: 475-481.

7. Lacroix M, Graiess F, Monnier-Cholley L, Arrivé L, 2020. SARSCoV-2 pulmonary infection revealed by subcutaneous emphysema and pneumomediastinum. Intensive Care Med 26: 1620-1621.

8. López Vega JM, Parra Gordo ML, Diez Tascón A, Ossaba Vélez S, 2020. Pneumomediastinum and spontaneous pneumothorax as an extrapulmonary complication of COVID-19 disease. Emerg Radiol, doi: 10.1007/s10140-020-01806-0.

9. Noppen M, 2010. Spontaneous pneumothorax: epidemiology, pathophysiology and cause. Eur Respir Rev 19: 217-219.

10. Sahni S, Verma S, Grullon J, Esquire A, Patel P, Talwar A, 2013. Spontaneous pneumomediastinum: time for consensus. N Am J Med Sci 5: 460-464.

11. Sahn S, Heffner J, 2000. Spontaneous pneumothorax. N Engl J Med 342: 868-874.

12. Dajer-Fadel WL, Argüero-Sánchez R, Ibarra-Pérez C, NavarroReynoso FP, 2014. Systematic review of spontaneous pneumomediastinum: a survey of 22 years' data. Asian Cardiovasc Thorac Ann 22: 997-1002.

13. Ai T, Yang Z, Hou H, Zhan C, Chen C, Lv W, Tao Q, Sun Z, Xia L, 2020. Correlation of chest CT and RT-PCR testing in coronavirus disease 2019 (COVID-19) in China: a report of 1014 cases. Radiology 296: E32-E40.

14. Pan $F$ et al., 2020. Time course of lung changes on chest CT during recovery from 2019 novel coronavirus (COVID-19) pneumonia. Radiology 7: 1-7.

15. Sun R, Liu H, Wang X, 2020. Mediastinal emphysema, giant bulla, and pneumothorax developed during the course of COVID-19 pneumonia. Korean J Radiol 21: 541-544.

16. Xiang $\mathrm{C}, \mathrm{Wu}$ G, 2020. SARS-CoV-2 pneumonia with subcutaneous emphysema, mediastinal emphysema, and pneumothorax: a case report. Medicine (Baltimore) 99: e20208. 
17. Mohan V, Tauseen RA, 2020. Spontaneous pneumomediastinum in COVID-19. BMJ Case Rep 13: 1-2.

18. Wang W, Gao R, Zheng Y, Jiang L, 2020. COVID-19 with spontaneous pneumothorax, pneumomediastinum and subcutaneous emphysema. J Travel Med, doi: 10.1093/jtm/taaa062.

19. Ucpinar BA, Sahin C, Yanc U, 2020. Spontaneous pneumothorax and subcutaneous emphysema in COVID-19 patient: case report. J Infect Public Health 13: 887-889.

20. Corrêa Neto IJF, Viana KF, da Silva MBS, da Silva LM, de Oliveira G, Cecchini ARdaS, Rolim AS, Robles L, 2020. Perforated acute abdomen in a patient with COVID-19: an atypical manifestation of the disease. $J$ Coloproctol 40: 269-272.

21. Hollingshead C, Hanrahan J, 2020. Spontaneous pneumothorax following COVID-19 pneumonia. IDCases 21: e00868.

22. Rohailla S, Ahmed N, Gough K, 2020. SARS-CoV-2 infection associated with spontaneous pneumothorax. CMAJ 192: E510.

23. Spiro JE, Sisovic S, Ockert B, Böcker W, Siebenbürger G, 2020. Secondary tension pneumothorax in a COVID-19 pneumonia patient: a case report. Infection, doi: 10.1007/s15010-020-01457-w.
24. Flower L, Carter JPL, Rosales Lopez J, Henry AM, 2020. Tension pneumothorax in a patient with COVID-19. BMJ Case Rep 13: 1-4.

25. Aiolfi A, Biraghi T, Montisci A, Bonitta G, Micheletto G, Donatelli F, Cirri S, Bona D, 2020. Management of persistent pneumothorax with thoracoscopy and blebs resection in COVID-19 patients. Ann Thorac Surg, doi: 10.1016/j.athoracsur.2020.04.011.

26. Falagas ME, Pitsouni El, Malietzis GA, Pappas G, 2008. Comparison of PubMed, Scopus, Web of Science, and Google Scholar: strengths and weaknesses. FASEB J 22: 338-342.

27. Kolani S, Houari N, Haloua M, Alaoui Lamrani Y, Boubbou M, Serraj M, Aamara B, Maaroufi M, Alami B, 2020. Spontaneous pneumomediastinum occurring in the SARS-COV-2 infection. IDCases 21: 4-7.

28. LeiP, Mao J, Wang P, 2020. Spontaneous pneumomediastinum ina patient with coronavirus disease 2019 pneumonia and the possible underlying mechanism. Korean J Radiol 21: 929-930.

29. Wang J, Su X, Zhang T, Zheng C, 2020. Spontaneous pneumomediastinum: a probable unusual complication of coronavirus disease 2019 (COVID-19) pneumonia. Korean J Radiol 21: 627-628. 